\title{
PERFORMANCE OPTIMIZATION OF RADIOACTIVE WASTE TRANSMUTATION IN ACCELERATOR DRIVEN SYSTEM
}

\author{
Anna Golovkina \\ Department of Control Systems \\ Theory for Electrophysical Facilities \\ Saint Petersburg State University \\ Russia \\ a.golovkina@spbu.ru
}

\author{
Dmitri Ovsyannikov \\ Department of Control Systems \\ Theory for Electrophysical Facilities \\ Saint Petersburg State University \\ Russia \\ d.a.ovsynnikov@spbu.ru
}

\author{
Sorin Olaru \\ Laboratoire des Signaux et Systémes \\ CentraleSupélec, \\ Université Paris Saclay \\ France \\ sorin.olaru@centralesupelec.fr
}

Article history:

Received 04.12.2018, Accepted 21.12.2018

\begin{abstract}
Long-lived actinides transmutation in accelerator driven system (ADS) is considered in this paper. The objective is to improve the transmutation performance from the overall radiotoxity of nuclear waste point of view by means of an optimization approach based on control theory. Parameters subjected to determination are the initial values of actinides nuclear concentration in the loaded fuel. The change in time of actinides' atomic concentration is described by a system of ordinary differential equations with initial conditions, that can be given with some inaccuracy. For such dynamical system a gradient-based optimization algorithm taking into account robustness of initial loading will be constructed. Moreover, constraint on value of effective multiplication factor is considered during numerical optimization. Based on this approach initial atomic concentration values providing the best indicators of actinides burnup can be obtained. Calculation results of actinides volume fraction and effective multiplication factor dynamics are presented.
\end{abstract}

\section{Key words}

Optimization, optimal control, ADS, transmutation, radioactive waste.

\section{Introduction}

The level of ecological impact of nuclear power engineering is measured by number of radionuclides (fission products (FP) and actinides (MA)), which are formed during nuclear reactors operation.

The radiotoxicity of spent nuclear fuel is firstly dominated by short-lived fission products, and later, by actinides [nuc, 1996]. A few hundred-thousand years after the discharge, the radiotoxicity of the unprocessed fuel drops to the natural toxicity level for traditional thermal reactors, i.e. the equilibrium radiotoxicity of the natural uranium ore [Mai, 2002]. The separation (and intermediate storage) of uranium and plutonium could reduce the radiotoxicity of the remaining highlevel radioactive waste by an order of magnitude, but it would still take about twenty-thousand years for the radiotoxicity of this waste to reach the natural toxicity level [oec, 2006].

Ecological impact of short-lived radionuclides can be reduced till the admissible level using organizational and technical measures in storage and fuel cycle facilities. However, in case of geological disposal, longlived nuclear waste has a potential risk over a few ten thousands years. The geological disposal site is difficult to select, mainly because it is hard to predict the changes in the structure of ground layers for the coming ten thousands years. Due to these facts, the problem of long-lived radioactive waste elimination arises, as an important technological research topic. The de- 
cision for this problem can find a pertinent solution in transmutation [Coquelet and et al, 2009].

Transmutation is a transformation of longed-lived radionuclides into short-lived or stable isotopes under influence of neutral or charged particle flows.

From the viewpoint of the radiotoxicity, transmutation must first be concerned with the minor actinides, particularly americium and neptunium, because the toxicity of the fission products lays at least two orders of magnitude below that of the actinides after a few hundred years [Mai, 2002].

For the transmutation of actinides, the key reaction is the fission reaction which transforms long-lived, highly radiotoxic actinides into mostly short-lived, less toxic fission products. Other reactions such as capture and $(n, 2 n)$ reactions just transform actinide species into other actinide species without a significant effect on long-term radiotoxicity [Tucek, 2004]. However, these reactions are useful insofar as they transform fertile actinides with a low fission probability into fissile actinides with a high fission probability.

Fast-spectrum systems are required to handle the minor actinides. Conventional fast reactors can burn selfgenerated minor actinides in a closed cycle, but they are not suited for burning pure minor actinides. In this regard accelerator-driven systems are considered to provide a promising way of actinides transmutation.

The concept of accelerator-driven systems (frequently called hybrid systems) combines a particle accelerator with a sub-critical core [Nifenecker et al., 2003]. The principal advantages that accelerator-driven subcritical systems have relative to critical reactors are twofold: greater flexibility with respect to fuel composition, and potentially enhanced safety. Accelerator driven systems are ideally suited to burning fuels which are problematic from the standpoint of critical reactor operation, namely, fuels that would degrade neutronic characteristics of the critical core to unacceptable levels due to small delayed neutron fractions and short neutron lifetimes [Golovkina, 2017]. It should also be noted that the proportionality of the reactor power to the accelerator current simplifies the reactor power maintenance [Golovkina et al., 2016].

In order to achieve better transmutation performance the initial fuel loading should be chosen in an optimal way. Most works devoted to this problem use combinatorial approach where a number of $x$ fuel assemblies are permuted in $l$ locations in the core [Rahman, 2015], [Washington and King, 2017], [Ishida and Sekimoto, 2010]. In this paper a different approach implementing optimization of MA composition in the fuel is proposed. This problem statement and model equations are presented in the section 2. Section 3 contains the description of optimization approach, with some calculations results placed in section 4 .

\section{Problem Statement}

Evolution of fuel concentration in ADS is described by a system of ordinary differential equations (also known as Bateman equations [Bateman, 1910]):

$$
\begin{aligned}
\frac{d N^{i}}{d t}= & \sum_{j \neq i} f^{j \rightarrow i} \lambda^{j} N^{j} \\
& +\sum_{k \neq i} \sum_{x} g_{x}^{k \rightarrow i} N^{k}\left\langle\sigma_{x}^{k} \phi\right\rangle+ \\
& +\sum_{l} \gamma^{l \rightarrow i} F^{l}-\left(\lambda^{i}+\left\langle\sigma_{a}^{k} \phi\right\rangle\right) N^{i}
\end{aligned}
$$

with given initial conditions:

$$
N^{i}(0)=N_{0}^{i}
$$

where

$N^{i}$ - atomic concentration of isotope $i$;

the first term on the right-hand side expresses the production rate of nuclide $i$ due to radioactive decay of another nuclide $j$ on the burnup chain;

$\lambda_{j}$ is the decay constant of nuclide $j$ and $f^{j \rightarrow i}$ is the probability of decay to nuclide $i$;

the second term is the production rate of nuclide $i$ due to the nuclear reaction $x$ of another nuclide $k$ the major nuclear reaction is the neutron capture reaction and other reactions such as the $(n, 2 n)$ reaction can be considered if necessary;

$g_{x}^{k \rightarrow i}$ is the probability of transmutation into nuclide $i$ for nuclear reaction $x$ of nuclide $k$;

the third term is for the production of FPs;

$F_{l}$ is the fission rate of heavy nuclide $l$ and $\gamma^{l \rightarrow i}$ is the production probability (yield fraction) of nuclide $i$ for the fission reaction;

the last term is the loss rate of nuclide $i$ due to radioactive decay and absorption reactions.

$\left\langle\sigma_{x}^{k} \phi\right\rangle$ - the microscopic reaction rate of nuclide $k$ integrated over all neutron energies — is defined by the following relation:

$$
\begin{aligned}
\left\langle\sigma_{x}^{k} \phi\right\rangle & =\int_{E_{\min }}^{E_{\max }} \sigma_{x}^{k}(E) \phi(E) d E \\
& \approx \sum_{i=1}^{G} \sigma_{x}^{k}\left(E_{i}\right) \phi\left(E_{i}\right) \Delta E_{i},
\end{aligned}
$$

where $\phi\left(E_{i}\right)$ represents the value of neutron flux normalized to the reactor heat power in the $i$-th energy group, $G$ - number of considered energy groups.

In general, neutron flux is a volume-averaged solution of stationary neutron transport equation. Its diffusion 


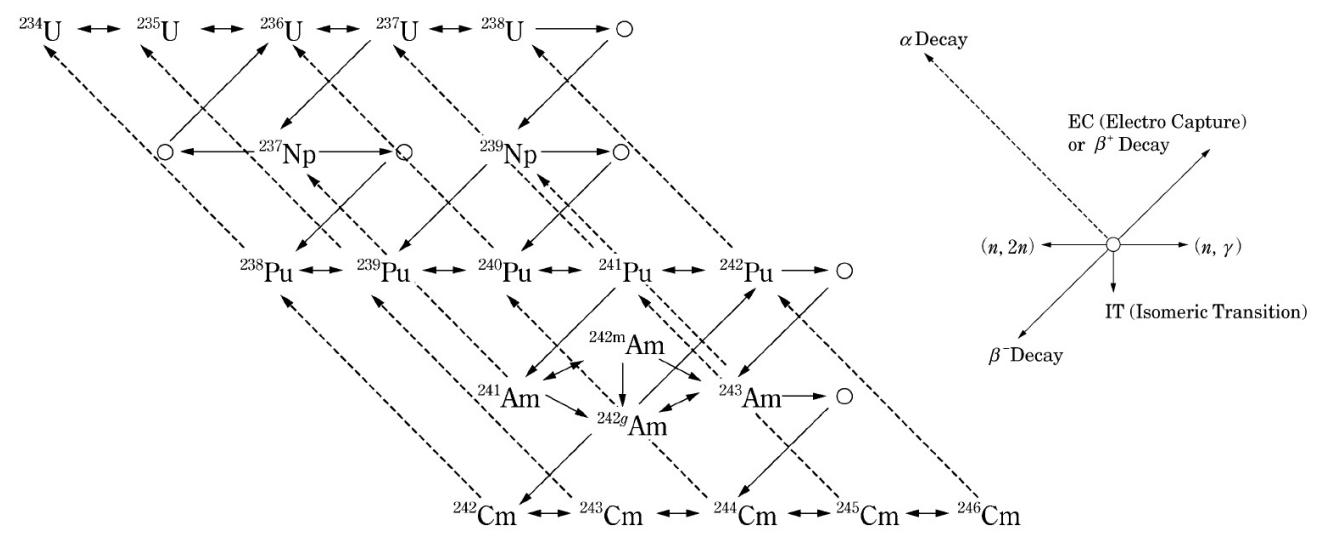

Figure 1. Relations of the most important actinides via different types of decay and neutron reactions

approximation will be considered in the present paper [Okumura et al., 2014]:

$$
D \nabla^{2} \phi-\Sigma_{a} \phi+S=0 .
$$

with the notation

$D$ - diffusion coefficient,

$\Sigma_{a}=\sum_{i=1}^{n} N^{i} \sigma_{a}^{i}-$ macroscopic absorption cross section in the core $(n-$ total number of considered isotopes),

$S$ - neutron supply from fission, scattering and external neutron source.

In should be noted that flux value in (1) is considered to be constant during the step of integration. Equation (4) is solved again with new atomic concentrations obtained after one step of (1) integration. Neutron flux from (4) is calculated in multi-group diffusion approximation using the program code MDSR [Golovkina and Kudinovich, 2017].

System (1) can be rewritten in the compact matrix form:

$$
\dot{x}=f(t, x)=A(t) x,
$$

where we assume that $x=\left[N^{1}, N^{2}, \ldots, N^{n}\right]$, $A(t)$ - matrix of $n \times n$ dimensions, constructed on the basis of burnup chain (see Fig. 1 [Okumura et al., 2014]) for considered actinides and $n$ is the number of considered isotopes [Golovkina et al., 2018b].

Initial conditions for system (5), determining the initial loading of fuel to the reactor core are given

$$
x(0)=\bar{x}_{0} .
$$

The aim of optimization process is to find $\bar{x}_{0}^{*}$ values providing the best performance of transmutation: minimal radiotoxity of discharged fuel. The initial values determining dynamics of system (5) can be effectively realized in the process with some error that can lead to the different performance of waste transmutation. In order to take into account such possibility it is supposed that initial values of nuclear concentrations $x_{i}(0)$ can be taken from some neighborhood of point $\bar{x}_{0 i}$. Then the initial values of nuclear concentrations can be taken from the interior part of the ellipsoid

$$
\left(x_{0}-\bar{x}_{0}\right)^{*} G_{0}\left(x_{0}-\bar{x}_{0}\right)=1,
$$

where $G_{0}$ is a positive definite symmetric matrix, $\bar{x}_{0}$ the ellipsoid center. Ellipsoid shift along the solutions of a linear system is also ellipsoid with center in $\bar{x}(t)$, bounding nuclear concentration initial values set in time moment $t$ :

$$
(x-\bar{x}(t))^{*} G(t)(x-\bar{x}(t))=1 .
$$

Here $\bar{x}(t)=x\left(t, \bar{x}_{0}\right)$ - the solution of system (5) with initial conditions (6), matrix $G(t)$ - determined by the solution of the following equation [Ovsyannikov, 1980]

$$
\frac{d G}{d t}=-A^{*}(t) G(t)-G(t) A(t)
$$

with initial conditions $G(0)=G_{0}$.

The inverse matrix $G^{-1}(t)$ satisfies the similar to (9) equation [Ovsyannikov, 1980], [Ovsyannikov, 1990]

$$
\frac{d G^{-1}}{d t}=A G^{-1}+G^{-1} A^{*}
$$

It's clear from the matrix equation (9) that initial condition (6) (considered here as a control actions) doesn't influence the matrix $G(t)$, determining the ellipsoid shape at some time moment $t$.

Because ADS is considered in the paper as transmutation facility, the requirement of its reactor to stay subcritical throughout the operation time should be taken 
into account. That means the effective multiplication factor should be less than one

$$
k_{\text {eff }}<1 \text {. }
$$

$k_{\text {eff }}$ is the lowest eigenvalue of translation operator and the corresponding eigenfunction $\varphi$ is neutron flux density in the reactor core [Vladimirov, 1971]. In this paper we use the diffusion approximation of translation equation in order to simplify calculations. In this case $k_{\text {eff }}$ is the lowest eigenvalue of equation (4), where $S=\frac{\nu \Sigma_{f}}{k_{\mathrm{eff}}} \varphi, \Sigma_{f}=\sum_{i=1}^{n} N^{i} \sigma_{f}^{i}-$ macroscopic fission cross section in the core $(n-$ total number of isotopes).

Condition (11) puts additional constraint on $\bar{x}\left(t, \bar{x}_{0}\right)$ values in time interval $[0, T]$.

\section{Optimization Approach}

The aim of optimization is to provide the minimal output waste radiotoxity after ADS operation ends in time $T$. The corresponding performance criterion in this case is

$$
J(p)=\sum_{i=1}^{n} \omega\left(\bar{x}_{i}^{2}(T)\right)
$$

where $\omega\left(\bar{x}_{i}^{2}(T)\right)=\bar{x}_{i}^{2}(T) \lambda_{i}$ - function, translating isotopes nuclear concentrations $\left[1 / \mathrm{cm}^{3}\right]$ in their specific radiotoxity $\left[\mathrm{Bq} / \mathrm{cm}^{3}\right], \lambda_{i}-$ radioactive decay constant $[1 / \mathrm{s}], p$ - control parameter subjected to optimization (in our case the initial values $\bar{x}_{0}$ ).

In this formulation the similar problem for advanced fuel cycle was solved in [Golovkina et al., 2018a]. In this paper it's proposed to consider the problem of robust optimization, stipulatory to the possible errors in fuel loading to the ADS reactor core.

In order to provide the robustness to the optimal solution besides minimization of (12) the problem of ellipsoid (8) radius constriction in moment $T$ should be taken into account.

Let us denote $S=G^{-1}, z=x-\bar{x}(t)$, then ellipsoid with matrix $S$ is described by the equation

$$
z^{*} S(t) z=1
$$

The objective is then to find the maximal value of $z$ on the ellipsoid (8) surface. Using the standard approach with Lagrangian multipliers we will get the following estimation [Ovsyannikov, 1990]

$$
\max _{z^{*} G(t) z=1} z_{i}=\sqrt{s_{i i}(t)}
$$

where $s_{i i}$ - diagonal elements of matrix $S, z_{i}$ - the $i$-th component of vector $z$. Thus functions $\sqrt{s_{i i}(t)}$ are the envelopes on the variables $x-\bar{x}(t)$ of system (5) [Ovsyannikov, 2012].

Thereby the optimized criterion, taking into account the system robustness is

$$
J(p)=\sum_{i=1}^{n} \omega\left(\bar{x}_{i}(T)+\sqrt{s_{i i}(T)}\right)^{2} .
$$

Gradient based optimization methods can be used to minimize functional (14). In this case the explicit expression of its gradient should be obtained

$$
\nabla J(p)=\frac{d J}{d p}=\left(\frac{\partial J}{\partial p_{1}}, \ldots \frac{\partial J}{\partial p_{n}}\right)^{*}
$$

Let us denote $y_{i}=\bar{x}_{i}(T)+\sqrt{s_{i i}(T)}, \varphi(\cdot)=$ $\sum \omega\left(\cdot{ }^{2}\right)$, then the expression (14) can be rewritten in the form

$$
J(p)=\sum_{i=1}^{n} \omega\left(y_{i}^{2}(T)\right)=\varphi(y(T)) .
$$

$$
\frac{d J}{d p}=\frac{d \varphi}{d y} \cdot \frac{d y}{d p}=\left(\frac{\partial \varphi}{\partial y_{1}} \ldots \frac{\partial \varphi}{\partial y_{n}}\right) \cdot\left(\frac{\partial y}{\partial p_{1}} \ldots \frac{\partial y}{\partial p_{n}}\right)^{*}
$$

$$
\frac{d y}{d p_{i}}=\frac{\partial \bar{x}_{i}(T)}{\partial p_{i}}+\frac{\partial \sqrt{s_{i i}(T)}}{\partial p_{i}}
$$

In the expression (16) the first term can be obtained using variation equation [Golovkina et al., 2018a]

$$
\begin{aligned}
& \frac{d}{d t}\left(\frac{\partial x(t, p)}{\partial p_{i}}\right)=A(t) \frac{\partial x(t, p)}{\partial p_{i}} \\
& \frac{\partial x(0, p)}{\partial p_{i}}=\mathbb{E}
\end{aligned}
$$

The second term in (16) is equal to zero, because as shown above in the case of dynamical process described by a linear system (5) the elements of matrix $S$ do not depend on the parameter $p$. Their values at each moment of time $t$ are completely determined by the initial values from $S_{0}$ and matrix of the system (5) $A$. Thus the robustness of initial loading (uncertainty limits of the parameter $p$ ) can be estimated by matrix $S_{0}$.

Taking into account the previous considerations gradient components can be calculated using the following formula

$$
\frac{\partial J}{\partial p_{i}}=2\left(\bar{x}_{i}(T)+\sqrt{s_{i i}}(T)\right) \frac{\partial \bar{x}_{i}(T)}{\partial p_{i}} .
$$


Thus optimization problem is narrowed down to the solution of equation (17) and the following use of one of the gradient-based methods constructed with the obtained expression for gradient components (18). It should be noted that taking into account the constraint (11) now is carried out in the following way. At each optimization step the value of (11) is computed, if it exceeds 1 then the step size in optimization method should be reduced. More comprehensive way of considering this condition is being developed.

\section{Calculation Results}

Program code for calculation of fuel isotopes composition dynamics and optimization of initial fuel loading is developed in order to implement the described techniques. It is shown that the burnup capability of ADS is better in comparison with conventional nuclear reactors. Transmutation performance criterion (14), taking into account robustness of the system is used as an objective function. As was described earlier control of constraints values (11) is carried out on each of the step during numerical optimization. Fig. 2 shows the evolution of the neutron multiplication factor during the operation time. In table 1 volume fractions of transmutated actinides in initial ADS loading and their fractions by the end of operation cycle are presented. Calculation also showed that overall radioactivity of the charged fuel was reduce in approximately 5 times.

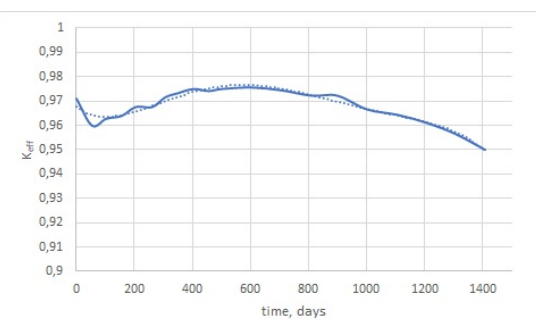

Figure 2. $k_{\text {eff }}$ time evolution during transmutation cycle (dotted line is an approximation curve)

\begin{tabular}{|l|l|l||l|l|l|}
\hline Isotope & $\begin{array}{l}\text { vol \% } \\
\text { before }\end{array}$ & $\begin{array}{l}\text { vol \% } \\
\text { after }\end{array}$ & Isotope & $\begin{array}{l}\text { vol \% } \\
\text { before }\end{array}$ & $\begin{array}{l}\text { vol } \% \\
\text { after }\end{array}$ \\
\hline $\mathrm{Pu}^{238}$ & 1.0 & 8.0 & $\mathrm{Am}^{241}$ & 12.0 & 6.0 \\
\hline $\mathrm{Pu}^{239}$ & 52.0 & 17.0 & $\mathrm{Am}^{243}$ & 0.2 & 4.0 \\
\hline $\mathrm{Pu}^{240}$ & 21.0 & 37.0 & $\mathrm{Cm}^{242}$ & 0.2 & 0.5 \\
\hline $\mathrm{Pu}^{241}$ & 2.0 & 6.0 & $\mathrm{Cm}^{243}$ & 0.2 & 0.5 \\
\hline $\mathrm{Pu}^{242}$ & 5.0 & 13.0 & $\mathrm{Cm}^{244}$ & 0.2 & 4.0 \\
\hline $\mathrm{Np}^{237}$ & 6.0 & 2.0 & $\mathrm{Cm}^{245}$ & 0.1 & 1.0 \\
\hline
\end{tabular}

Table 1. Comparison of actinides volume fraction in charged and discharged fuel in ADS

\section{Conclusion}

Optimization approach based on control theory applied to the problem of long-lived actinides transmutation in accelerator driven system is considered in the paper. The transmutation performance criterion taking into account the system robustness is constructed. Gradient-based optimization algorithm is designed and implemented as a program tool. Constraint on value of effective multiplication factor is taken into account during numerical optimization. Based on this approach some calculations results were obtained. After optimization radioactivity of fuel charged in the core was reduced in approximately 5 times.

\section{References}

(1996). Nuclear Wastes: Technologies for Separations and Transmutation. The National Academies Press, Washington, DC.

(2002). Accelerator-driven systems (ads) and fast reactors (fr) in advanced nuclear fuel cycles. Technical report, OECD Nuclear Energy Agency.

(2006). Physics and safety of transmutation systems. a status report. Technical Report 6090, OECD, Paris, France.

Bateman, H. (1910). Solution of a system of differential equations occurring in the theory of radioactive transformations. Proc. Cambridge Philos. Soc., 15:423-427.

Coquelet, C. and et al (2009). Comparison of different options for transmutation scenarios studied in the frame of the french law for waste management. Revue Générale Nucléaire, (6):73-82.

Golovkina, A. (2017). Simplified dynamics model for subcritical reactor controlled by linear accelerator. Cybernetics and Physics, 6(4):201-207.

Golovkina, A. and Kudinovich, I. (2017). Program for multi-group diffusion calculation of subcritical reactor neutron-physical characteristics (mdsr).

Golovkina, A., Kudinovich, I., and Ovsyannikov, D. (2018a). Optimization model for radioactive waste transmutation in advanced fuel cycle. In 17th IFAC Workshop on Control Applications of Optimization, pages 179-183, Yekaterinburg, Russia.

Golovkina, A., Kudinovich, I., and Svistunov, Y. (2016). The problem of ads power-level maintenance. Cybernetics and Physics, 5(2):52-58.

Golovkina, A. G., Loskutova, A. D., and Ovsyannikov, D. A. (2018b). Nuclear constants database for fuel isotope change estimation. Problems of atomic science and technology, 115(3):159-162.

Ishida, S. and Sekimoto, H. (2010). Finding the best fuel assemblies shuffling scheme of ads for ma transmutation using dynamic programming. Nuclear Engineering and Design, 240:3645-3653. 
Nifenecker, H., Meplan, O., and David, S. (2003). Accelerator Driven Subcritical Reactors. Institute of Physics Publishing Bristol and Philadelphia.

Okumura, K., Oka, Y., and Ishiwatari, Y. (2014). Nuclear reactor design, chapter 2, pages 49-126. Springer, London.

Ovsyannikov, D. A. (1980). Mathematical methods of beam control. Leningrad University. (in Russian).

Ovsyannikov, D. A. (1990). Modeling and optimization of charged particle beam dynamics. Leningrad University, Leningrad. (in Russian).

Ovsyannikov, D. A. (2012). Mathematical modeling and optimization of beam dynamics in accelerators. In Proceedings of 23rd Russian Particle Accelerator Conference, pages 68-72, Saint Petersburg, Russia.
Rahman, F. B. A. (2015). Deterministic methods for multi-control fuel loading optimization. $\mathrm{PhD}$ thesis, Nuclear Engineering and Radiological Sciences (University of Michigan).

Tucek, K. (2004). Neutronic and Burnup Studies of Accelerator-driven Systems Dedicated to Nuclear Waste Transmutation. PhD thesis, Stockholm Royal Institute of Technology.

Vladimirov, V. S. (1971). Equations of Mathematical Physics. Marcel Dekker.

Washington, J. and King, J. (2017). Optimization of plutonium and minor actinide transmutation in an ap1000 fuel assembly via a genetic search algorithm. Nuclear Engineering and Design, 311:199-212. 